\title{
Throughput and QoS Pricing in Wireless Communication
}

\author{
Andrey Garnaev \\ St. Petersburg State University \\ St. Petersburg, Russia \\ garnaev@yahoo.com
}

\author{
Yezekael Hayel \\ University of Avignon, France \\ Avignon, France \\ yezekael.hayel@univ- \\ avignon.fr
}

\author{
Konstantin Avrachenkov, \\ Eitan Altman \\ INRIA Sophia Antipolis, \\ France \\ Sophia Antipolis, France \\ k.avrachenkov@sophia.inria.fr \\ altman@sophia.inria.fr
}

\begin{abstract}
In this paper we investigate a bi-level economic model of wireless communications. We take into account the user's demand and also the QoS offered by the network. We determine with our model when a provider can make more profit by investing into new technology. We consider a Stackelberg game approach where the provider decides the price per rate. In fact, users have to pay for the throughput they have contracted with the service provider in their SLA. Then, pricing for throughput is more realistic then pricing for power as it is suggested in several papers in the literature. Our approach is general as we have important results for general user's utility function with only natural assumptions on them. We also study next an incomplete information case in which the provider has no perfect information about the user demands in network and its quality. To deal with it we apply a Bayesian approach. We demonstrate that the expectation on the increasing user demand can induce jumping reduction of the optimal tariff meanwhile impairs expectation about increasing in user activity can lead to jumping increasing in the optimal tariff. This jumping nature of tariff tells about importance of marketing research for provider to correctly estimate user's demand in his intention of using network and how he personally values the service. Finally, we consider the multiuser case and obtain a closed form expression of the Stackelberg equilibrium in that case. In particular we show that the provider cannot essentially increase its profit by simple increasing number of users.
\end{abstract}

\section{INTRODUCTION}

In this paper we study pricing mechanism based on user's capability, quality of the network and the user's demand to such quality. We also consider how incomplete information about user's and mutual interference of user's impacts this problem. Along with investigation these plots we suggest an answer to the question when it is reasonable for a provider to invest into new technology to improve the quality of service. To deal with all these questions we introduce a Stackelberg

Permission to make digital or hard copies of all or part of this work for Permission to make digital or hard copies of all or part of this work for personal or classroom use is granted without fee provided that copies are not made or distributed for profit or commercial advantage and that copies bear this notice and the full citation on the first page. To copy otherwise, to republish, to post on servers or to redistribute to lists, requires prior specific permission and/or a fee. VALUETOOLS 2011, May 16-20, Paris, France Copyright (C) 2011 ICST 978-1-936968-09-1 DOI 10.4108/icst.valuetools.2011.245699 game for throughput pricing. Why pricing for throughput? First we would like to note that Stackelberg game approach is very popular among researchers dealing with pricing in networks (see, for example, [6, 3, 4, 5, 10, 7, 9, 8, 11]). All these papers deal with power pricing. In this paper we introduce the other kind of pricing, throughput pricing, which measures the transmitted information per second in wireless network and so, it could serve as a simple measure for using network and charging for it the users by provider. Namely, in this paper we consider a tariff game between a provider and a user or many users. Of course the value of the tariff has an impact on user's behaviour. If tariff is small then user tends to exploit the network as much as possible or even abuse the network. On the other hand large tariff can cut user off the network at all. A natural question arises for the provider: which tariff the provider has to assign to get the maximal profit. This situation is modelled by a Stackelberg game with provider as a leader and a user or users as followers. We consider that the payment of the user to the provider is proportional to the throughput. The user capability is measured by the total power the user could use for transmission. The quality of the network is presented by the fading channel gain and noise interference. The user's demand on the quality of the network is presented by utility of his throughput. In multi-user plot different users can have different utilities. Through different utility functions several QoS requirements can be modelled.

We deal with the Stackelberg game in three scenarios. First, as basic one, we study the particular case with one user and a provider game. The second one is the case where the provider does not have a complete information on the user's demand. This happens in the real world where the provider has only some statistic data. To deal with this plot, we apply Bayesian equilibrium approach which allows us to find a threshold value telling the provider which possible demand has to be satisfied in full, and which has to be satisfied only partially. Finally, we consider multi-user scenario where user can have either the same or different utilities like different class of users.

\subsection{Organization of the paper}

The rest of this paper is organized as follows. In Section 2, we describe the system model with a user and a provider, state the assumptions, and give the mathematical formulation for the problem under consideration. Also, we supply the solution of the problem and result of numerical modelling. In Section 3, we treat a generalization of the problem for the case where the total power is unknown by 
the provider. In Section 4 we develop our model for the case of one provider and $N$ users case with different and equal user utilities. In Section 5 discussions of the obtained result aregiven.

\section{ONE PROVIDER AND ONE USER}

In this section we consider the following basic tariff game which will be generalized in the next sections. A user intents to use a network for transmission a signal. We consider that there is no externalities cost, i.e. the mobiles do not interfere each other. The network is not a public one so user has to pay for its usage. The tariff is charged to the user based on the attained throughput. A natural question arises for provider: which tariff the provider has to assign to get the maximal profit. This situation can be modeled by a Stackelberg game with provider as a leader and a user as a follower, so as two steps game where

- on the first step for a fixed tariff, users decide in which size to exploit the network,

- on the second step the provider decides, knowing user's behaviour, what tariff to assign to get the maximal profit.

Those steps are described here to explain how we compute the best action for the provider (the leader of the game). It has no link with the sequence of the players in a stackelberg game.

\subsection{Stackelberg game}

We assume that the payment of user is proportional to his throughput, so the provider assigns tariff $C$ per unit of user's throughput.

On the first step of the Stackelberg game the tariff $C$ is fixed. It is natural to consider that the strategy of the user is the transmitted power $T \in[0, \bar{T}]$ with $\bar{T}$ is the maximal power the user has in his mind to apply to. The user's payoff is the difference between his satisfaction of the network service which is an utility $U$ on his throughput and how much it costs him, i.e.

$$
v(T)=U(R(T))-C R(T),
$$

where $R=R(T)$ is the throughput which can be approximated valued as the Shannon capacity, i.e. $\ln (1+h T)$. We consider several natural assumptions on the utility function mostly considered in wireless network.

- $U(x)$ is strictly increasing and positive in $(0, \infty)$,

- $U^{\prime}(x)$ is strictly decreasing,

- $U(0)=0, U(+\infty)=+\infty, U^{\prime}(0+)>0$ and let $\bar{C}=$ $\sup _{U^{\prime}(C)>0} C$.

As examples of the user utility, it is reasonable to consider either a logarithmic one, or $\alpha$-fairness or shifted $\alpha$-fairness:

$$
\log (1+x), \quad \frac{1}{1-\alpha} x^{1-\alpha}, \quad \frac{1}{1-\alpha}\left((1+x)^{1-\alpha}-1\right)
$$

with $\alpha \in(0,1)$. Note that the utility $\frac{1}{1-\alpha}\left((1+x)^{1-\alpha}-1\right)$ called the shifted SINR in [2]. It was introduced for an $\alpha$-fairness problem in order to joint in the same scale the
SINR's utility $(\alpha=0)$ and the Shannon capacity $(\alpha=1)$. It is clear that $\bar{C}=1$ for logarithmic and shifted $\alpha$-fairness utilities and $\bar{C}=\infty$ for $\alpha$-fairness utility. Also note that the throughput $R(T)$ also is a strictly increasing function such that $R(0)=0$.

In this section we assume that the maximal signal $\bar{T}$ and the fading channel gains $h$ are known to the user.

On the second step of the Stackelberg game, the provider looks after the optimal tariff $C$ in order to maximize its payoff which is given as follows:

$$
v_{P}(C)=C R(T(C)) .
$$

\subsection{Optimal strategies}

Now, we look first for the optimal power strategy of the user depending on the price of the provider. Second, given that reaction function, the best-response of the follower, we compute the optimal price $C$ determine by the provider in order to optimize his payoff. In order to find the optimal user strategy, we note that

$$
\frac{d v}{d T}=\left(U^{\prime}(R(T))-C\right) R^{\prime}(T)
$$

Thus, the optimal user strategy is defined by

$$
T(C)=\min \left\{\left[R^{-1}\left(\left(U^{\prime}\right)^{(-1)}(C)\right)\right]_{+}, \bar{T}\right\}
$$

which is equivalent to

$$
T(C)= \begin{cases}0, & U^{\prime}(0) \leq C \\ R^{-1}\left(\left(U^{\prime}\right)^{(-1)}(C)\right), & U^{\prime}(R(\bar{T}))<C<U^{\prime}(0) \\ \bar{T}, & C \leq U^{\prime}(R(\bar{T})) .\end{cases}
$$

Now we have to find the optimal tariff $C$ from the provider point of view. The payoff of the provider is given by $v_{P}(C)=$ $C R(T(C))$. Then, by (3), we have

$$
v_{P}(C)= \begin{cases}0, & C \in I_{0} \\ C\left(U^{\prime}\right)^{(-1)}(C), & C \in I_{1} \\ C R(\bar{T}), & C \in I_{2},\end{cases}
$$

where

$$
I_{0}=\left[U^{\prime}(0), \infty\right), \quad I_{1}=\left(U^{\prime}(R(\bar{T})), U^{\prime}(0)\right)
$$

and

$$
I_{2}=\left[0, U^{\prime}(R(\bar{T}))\right] .
$$

It is clear that $v_{P}(C)$ is increasing on the interval $I_{2}$. Then we can deduce the following result on the optimal tariff of the provider.

THEOREM 1. The optimal tariff of the provider in the Stackelberg tariff game is

$$
C_{*}=\operatorname{argmax}_{C \in\left[U^{\prime}(R(\bar{T})), U^{\prime}(0)\right]} C\left(U^{\prime}\right)^{(-1)}(C) .
$$

Proof: Depending on the tariff $C$, the payoff of the provider is first increasing on the interval $I_{2}$ and finally constant on the interval $I_{0}$. Then, as it is a continuous function, it attains his maximum at:

$$
C_{*}=\operatorname{argmax}_{C \in I_{1}} C\left(U^{\prime}\right)^{(-1)}(C),
$$

where $I_{1}=\left[U^{\prime}(R(\bar{T})), U^{\prime}(0)\right]$. 
For the particular cases of the $\alpha$-fairness functions we have a more precise result which follows:

THEOREM 2. The optimal tariff $C_{*}$ of the service provider, when the utility functions are $\alpha$-fairness, is

$$
C_{*}=U^{\prime}(R(\bar{T}))
$$

which brings to the provider the total profit

$$
v_{P}=U^{\prime}(R(\bar{T})) R(\bar{T}) .
$$

Proof: We consider the $\alpha$-fairness utility functions depicted in (2):

$$
\frac{1}{1-\alpha} x^{1-\alpha} \text { and } \frac{1}{1-\alpha}\left((1+x)^{1-\alpha}-1\right) .
$$

(i) For $U(x)=\frac{1}{1-\alpha} x^{1-\alpha}$ we have that for $C \in I_{1}$ the user's optimal strategy and the provider's payoff are given as follows:

$$
\begin{aligned}
T(C) & =\frac{\exp \left(C^{-1 / \alpha}\right)-1}{h}, \\
v_{P}(C) & =C^{1-1 / \alpha}
\end{aligned}
$$

where

$$
I_{1}=\left[\frac{1}{\ln ^{\alpha}(1+h \bar{T})}, \infty\right) .
$$

Since $0<\alpha<1, v_{P}(C)$ is decreasing in $I_{1}$.

(ii) for $U(x)=\frac{1}{1-\alpha}\left((1+x)^{1-\alpha}-1\right)$ and so, as a particular case with $\alpha=1$, for $U(x)=\log (1+x)$, we have that for $C \in I_{1}$ the user's optimal strategy and the provider's payoff are given as follows:

$$
\begin{aligned}
T(C) & =\frac{\exp (1 / C-1)-1}{h}, \\
v_{P}(C) & =C^{1-1 / \alpha}-C,
\end{aligned}
$$

where

$$
I_{1}=\left[\frac{1}{(1+\ln (1+h \bar{T}))^{\alpha}}, 1\right] .
$$

Note that

$$
\frac{d v_{P}(C)}{d C}=\frac{\alpha-1}{\alpha} C^{-1 / \alpha}-1<0 \text { for } C<1 .
$$

Thus, $v_{P}(C)$ is decreasing in $I_{1}$.

So, we have proved that the payoff of the service provider is stricly decreasing over the interval $I_{1}$. Then, the optimal tariff $C_{*}$ of the service provider is given by:

$$
C_{*}=U^{\prime}(R(\bar{T})) \text {. }
$$

\subsection{Numerical illustrations}

In this section we perform some numerical illustrations of the previous results for the optimal tariff of the service provider.

- For shifted $\alpha$-fairness utility with $\alpha=0.1,0.5$ and 1 (so, for logarithmic utility also, since shifted $\alpha$-fairness utility coincides with logarithmic one for $\alpha=1$ ). Increasing $\alpha$ means that the user is less demandable to the network quality since he assigns larger value in his utility to the same throughput.

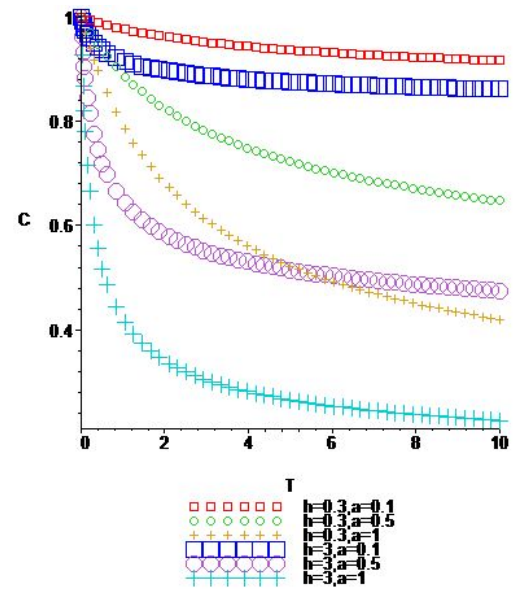

Figure 1: The optimal tariff $C$

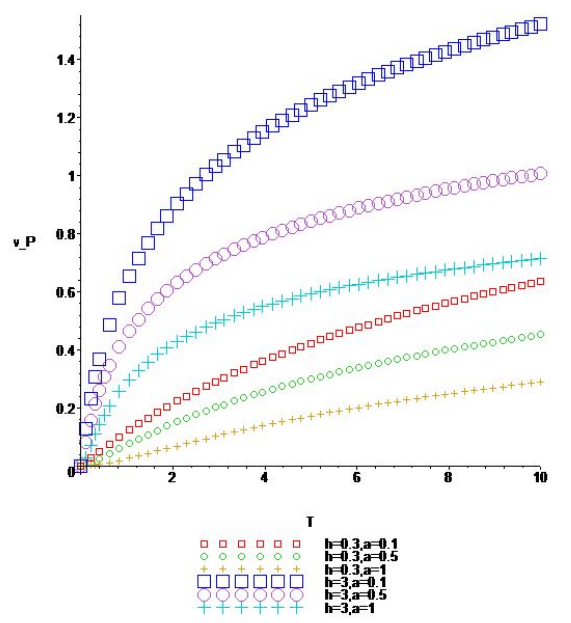

Figure 2: The optimal user payoff $v_{P}$

- For two values of fading channel gain $h=0.3$ and 3 . Bigger fading channel gain means that the network has better quality.

- For the total power $\bar{T}$ evaluating from 0 to 10 . The larger total power $\bar{T}$ means larger capability of the user to consume the network service. Why just intention? Because high tariff can reduce his real consuming of the network service.

The results of the numerical modelling are given on Figure 1 (for the optimal tariff) and Figure 2 (for the optimal provider profit).

- It shows that the less demandable user (with bigger $\alpha$ ) rises up tariff and the provider's profit.

- It demonstrates the improving quality of the network puts down up tariff and increasing the provider's profit. So, investing into new technologies that can improve service are always good for the provides as well as to 
the user. Of course, there is a question what cost of the technology has to be so that it would be profitable for provider to install it. Figure 1 also gives answer on this question. Say, the cost of the new technology is 0.2 . Then for the utility with $\alpha=0.1$ it is reasonable for provider to move to the new technology when user intents to employ the network with $\bar{T}=6$ since for such $\bar{T}=6$ the providers profit is 0.2 which can covers his corresponding expenses.

- It shows that bigger intention to employ the network $(\bar{T})$ by user cuts down tariff and increases the provider's profit.

Finally, we note that there exists an important difference between $\alpha$-fairness utility from one hand and logarithmic and shifted $\alpha$-fairness utilities from the other hand. Namely, $\alpha$-fairness assumes that if the user is not going to use the network (so, $\bar{T} \rightarrow 0$ ) then the tariff tends to infinity, meanwhile for logarithmic and shifted $\alpha$-fairness utilities it tends to 1 . Then it makes those utility functions more realistic and it also supplies extra motivation for employing shifted $\alpha$-fairness utility.

\section{INCOMPLETE INFORMATION}

In this section we consider the plot where the provider does not know the total power (so. user's capability) which user intents to transmit as well as his utility function but just their distribution. We investigate discrete distribution case, namely, the provider knows that with probability $q_{m r}$ the user's utility is $U_{m}$ and total user power is $\bar{T}_{m r}$ where $m \in$ $[1, M], r \in\left[1, M_{r}\right]$. Of course user knows which power and utility he has. To deal with this situation, we introduce $\bar{M}=\sum_{m=1}^{M} M_{m}$ types of user indexed by two numbers $m$ (index of user's utility) and $r$ (index of user's total power), i.e. user has type $r m$ if the total power to transmit is $\bar{T}_{m r}$ and his utility is $U_{m}$. Thus, we have the following results.

THEOREM 3. The optimal tariff of provider in the Stackelberg tariff game with incomplete information on total user power is given as follows

$$
C_{*}=\operatorname{argmax}_{C \in\left[0, \max _{r} U_{r}^{\prime}(0)\right]} \theta(C),
$$

where

$$
\theta(C)=C \sum_{m=1}^{M} \theta_{m}(C)
$$

and

$$
\begin{aligned}
& \theta_{m}(C)= \\
& = \begin{cases}\sum_{r=1}^{M_{m}} q_{m r} R\left(\bar{T}_{m r}\right), & C<U_{m}^{\prime}\left(R\left(\bar{T}_{m M_{m}}\right)\right), \\
\sum_{r=1}^{k-1} q_{m r} R\left(\bar{T}_{m r}\right) & \\
+\left(U_{m}^{\prime}\right)^{(-1)}(C) \sum_{r=k}^{M_{m}} q_{m r}, & U_{m}^{\prime}\left(R\left(\bar{T}_{m k}\right)\right)<C \\
& \text { and } C<U^{\prime}\left(R\left(\bar{T}_{m, k-1}\right)\right) \\
& \text { with } k \in\left[2, M_{m}\right] \\
\left(U_{m}^{\prime}\right)^{(-1)}(C), & U_{m}^{\prime}\left(R\left(\bar{T}_{m 1}\right)\right)<C \\
0, & \text { and } C<U_{m}^{\prime}(0), \\
0, & U_{m}^{\prime}(0) \leq C .\end{cases}
\end{aligned}
$$

Proof: On the first stage of the Stackelberg tariff game with incomplete information, the payoff to user of type $m r$ is given as follows:

$$
v_{m r}\left(T_{m r}\right)=U_{m}\left(R\left(T_{m r}\right)\right)-C R\left(T_{m r}\right) .
$$

On the second stage of the game the provider is going to maximize its expected profit, and so, its payoff is given as follows

$$
v_{P}(C)=C \sum_{m=1}^{M} \sum_{r=1}^{M_{m}} q_{m r} R\left(T_{m r}\right)
$$

By the previous section the optimal strategy $T^{r}=T^{r}(C)$ of user of type $r$ on the first stage as function on tariff is:

$$
T_{m r}(C)= \begin{cases}0, & U_{m}^{\prime}(0) \leq C \\ R^{-1}\left(\left(U_{m}^{\prime}\right)^{(-1)}\right)(C), & U_{m}^{\prime}\left(R\left(\bar{T}_{m r}\right)\right)<C \\ & \text { and } C<U_{m}^{\prime}(0) \\ \bar{T}_{m r}, & C \leq U^{\prime}\left(R\left(\bar{T}_{m r}\right)\right) .\end{cases}
$$

Without loss of generality we can renumber the user of type $m r$ in non-increasing order by $r$ :

$$
\bar{T}_{m 1} \geq \bar{T}_{m 2} \geq \ldots \geq \bar{T}_{m M_{m}} .
$$

Then the expected profit of the provider can be obtained as a function of tariff $C$ as follows:

$$
v_{P}(C)=C \sum_{m=1}^{M} \sum_{r=1}^{M_{m}} q_{m r} R\left(T_{m r}(C)\right)=C \sum_{m=1}^{M} \theta_{m}(C),
$$

where $\theta_{m}(C)$ is given by (5).

\subsection{A particular case: logarithmic utility}

In this section we find in closed form the optimal tariff for a particular case where each type of user has the same logarithmic utility $U(x)=\ln (1+x)$. Then we have $M$ types of user such that the user of type $i$ intends to use total power $\bar{T}_{i}$. Without loss of generality we can renumber the user of type $i$ in non-increasing order by $r$ :

$$
\bar{T}_{1} \geq \bar{T}_{2} \geq \ldots \geq \bar{T}_{M} .
$$

Then we have the following result on the optimal tariff of the service provider.

THEOREM 4. Let all user's type have the same logarithmic utility. Then the optimal tariff $C_{*}$ is given as follows:

$$
C_{*}=\frac{1}{1+\ln \left(1+h \bar{T}_{k_{*}}\right)},
$$

where $k_{*}$ is the unique integer such that

$$
\varphi_{k_{*}}<0<\varphi_{k_{*}+1},
$$

and

$$
\varphi_{k}=\sum_{r=1}^{k-1} q_{r} R\left(\bar{T}_{r}\right)-\sum_{r=k}^{M} q_{r}
$$

Proof: Then, since $C\left(U^{\prime}\right)^{(-1)}(C)=1-C$, by Theorem 3 the optimal expected profit of the provider as function of tariff $C$ is given as follows:

$$
v_{P}(C)= \begin{cases}\varphi_{k} C-\sum_{r=k}^{M} q_{r}, & d_{k} \leq C<d_{k-1}, \\ 0, & k \in[1, M+1], \\ & C \geq 1 .\end{cases}
$$


where

$$
\varphi_{k}=\sum_{r=1}^{k-1} q_{r} R\left(\bar{T}_{r}\right)-\sum_{r=k}^{M} q_{r}
$$

and

$$
d_{r}=\frac{1}{1+\ln \left(1+h \bar{T}_{r}\right)}
$$

with $\bar{T}_{M+1}=0$ and $\bar{T}_{0}=\infty$.

Note that, $\left\{\varphi_{k}\right\}$ is increasing sequence since

$$
\varphi_{k+1}-\varphi_{k}=q_{k}\left(R\left(\bar{T}_{k}\right)+1\right)>0 .
$$

Also,

$$
\varphi_{1}=-\sum_{r=1}^{M} q_{r}<0, \quad \varphi_{M+1}=\sum_{r=1}^{M} q_{r} R\left(\bar{T}_{r}\right) .
$$

Then, since $v_{P}(C)$ is increasing (decreasing) in $\left[d_{k}, d_{k-1}\right]$ with positive (negative) $\varphi_{k}$ we have the following result supplying the optimal tariff $C_{*}$ in closed form given by (7) with $k_{*}$ is the unique integer given by (8).

\subsection{Numerical illustration for the incomplete information}

In this section we perform some numerical illustrations which demonstrate that the optimal tariff and the expected provider profit can have jumps since $\theta(C)$ has switching points $\left\{p_{m k}=\right.$ $\left.U_{m}^{\prime}\left(R\left(\bar{T}_{m k}\right)\right)\right\}$. Its derivative has jumps and switching point $p_{m k}$ does not depend on the probabilities $\left\{q_{m k}\right\}$ and depends only on the total power $\bar{T}_{m k}$. So, variation of $\left\{q_{m k}\right\}$ and $\bar{T}_{m k}$ can cause jumps in the optimal tariff.

As numerical examples we deal with two values of fading channel gain $h=0.3$ and 3 (bigger fading channel gain means that the network has better quality) and the same and different user utilities separately.

(a) The same utility for all the user types. So, $M=1$ and let $M_{1}=2$.

Figures 3 and 4 illustrate how the optimal tariff and the expected optimal provider profit evaluate while total power $\bar{T}_{2}:=\bar{T}_{12}$ increases from 1 to 3 . We assume that total power $\bar{T}_{1}:=\bar{T}_{11}$ is fixed and equals to 1 , and $\bar{T}_{1}$ and $\bar{T}_{2}$ take place with probabilities $q_{1}:=q_{11}=0.2$ and $q_{2}:=q_{12}=0.8$ respectively. We deal here with shifted $\alpha$-fairness utility where $\alpha=0.1$ and 1 (so, for logarithmic utility also, since shifted $\alpha$-fairness utility coincides with logarithmic one for $\alpha=1$ ). Expectation that user is going to consume more provider's service leads to decreasing in tariff and decreasing in expected provider profit but it happens not so fast as in case with complete information.

Meanwhile for a fixed $\bar{T}_{1}=1$ and $\bar{T}_{2}=3$, figures 5 and 6 illustrate how the optimal tariff and the expected optimal provider profit evaluate while $q_{1}:=q_{11} \in[0,1]$. It shows that impairs expectation about increasing in user activity (so, when $q_{1}$ is increasing, and so, $q_{12}=1-q_{11}$ is decreasing) can lead to jumping increasing in tariff and decreasing expected provider profit, but of course the expected profit does it in continuous way.

(b) Different utility functions for all the users. Let $M=2$ and $M_{1}=M_{2}$. Also we assume that $\bar{T}_{11}=\bar{T}_{21}=1$ or 3 . We deal here with two shifted $\alpha$-fairness utility where
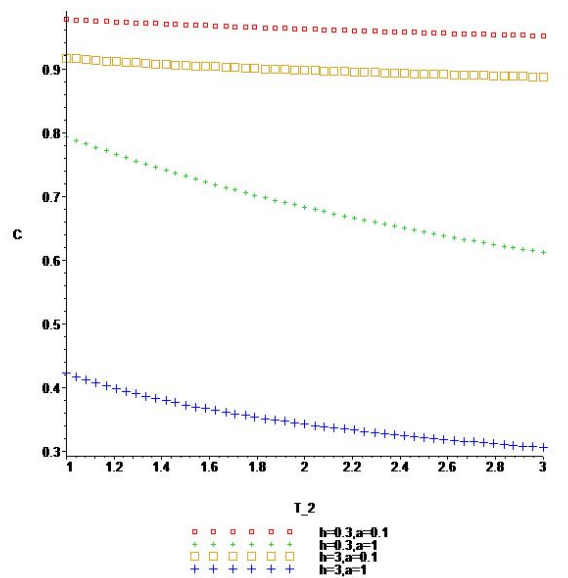

Figure 3: The optimal tariff for $T_{2} \in[1,3]$.

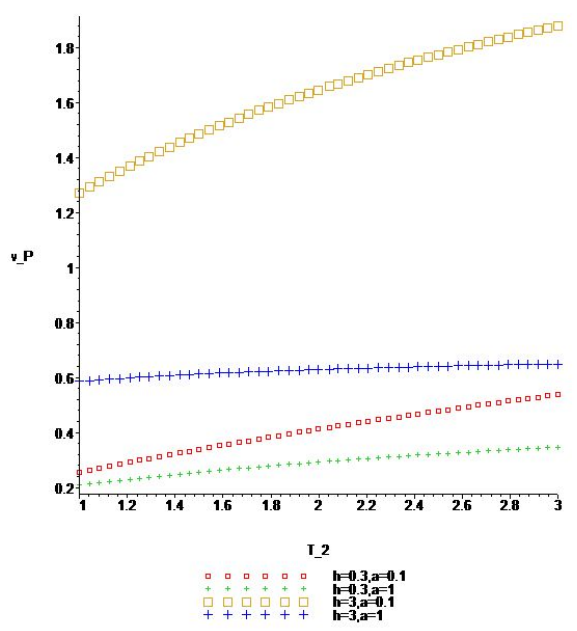

Figure 4: The expected optimal provider profit for $T_{2} \in[1,3]$.

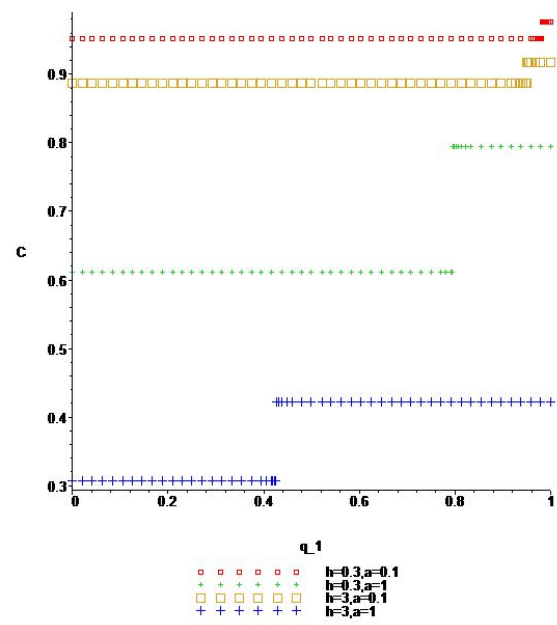

Figure 5: The optimal tariff for $q_{1} \in[0,1]$. 


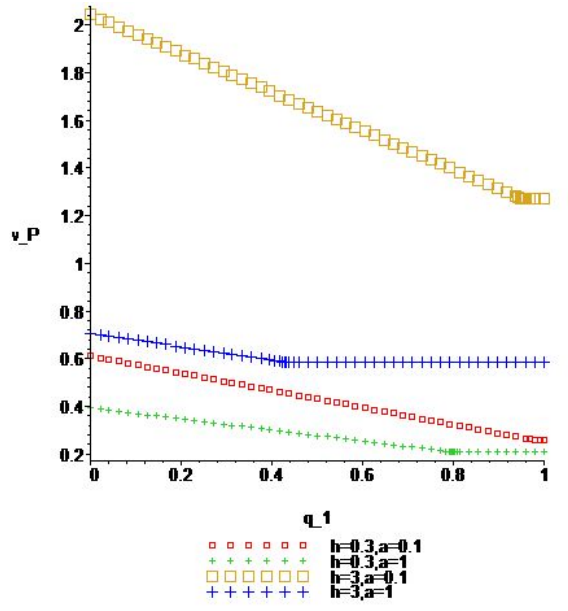

Figure 6: The expected optimal provider profit for $q_{1} \in[0,1]$.

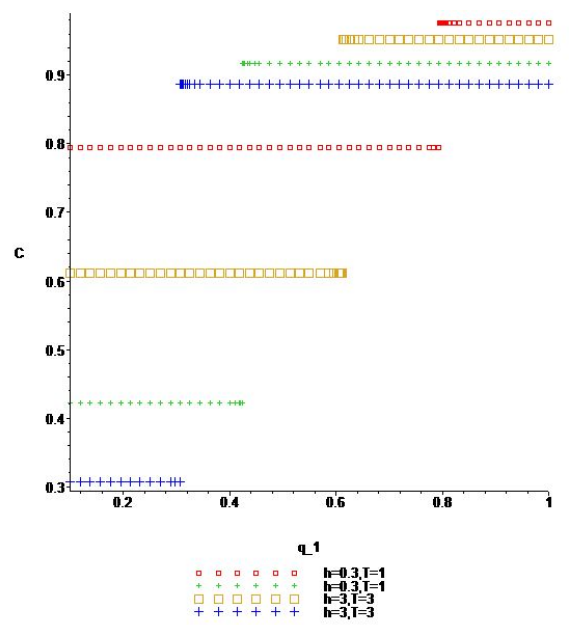

Figure 7: The optimal tariff for $\alpha_{1}=0.1$ and $\alpha_{2}=1$.

$\alpha=0.1$ and 1 which can take place with probability $q_{1}$ and $q_{2}=1-q_{1}$ respectively

Figures 8 and 9 show how the optimal tariff and the expected optimal provider profit evaluate while $q_{1}$ increases from 0 to 1 , and so, $q_{2}$ decreases from 1 to 0 . It shows that expectation about that the user is less demandable (so, $\alpha$ is bigger) could lead to increasing in tariff jumpily and increasing in expected provider profit.

This jumping nature of tariff tells about importance of marketing politics for provider to correctly estimate user's demand in exploiting of network and how he estimate useableness of the service.

\section{HETEROGENOUS USERS}

In this last section we consider heterogenous users case, namely, we assume that in network $N$ users and each user $i$ would like to transmit signal with a power $T_{i}$ where $T_{i} \geq 0$. Moreover, we do not assume that there is an upper bound

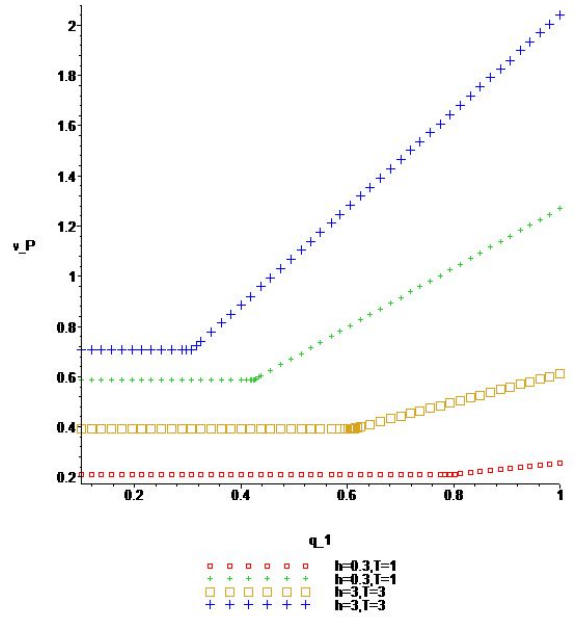

Figure 8: The expected optimal provider profit for $\alpha_{1}=0.1$ and $\alpha_{2}=1$

on the restriction transmitting signals. As throughput of user $i$ we consider the Shannon capacity, i.e.

$$
R_{i}(\mathbf{T})=\ln \left(1+\frac{L h_{i} T_{i}}{\sigma^{2}+\sum_{k=1, k \neq i}^{N} h_{k} T_{k}}\right),
$$

where

- $\mathbf{T}=\left(T_{1}, \ldots, T_{N}\right)$ is the user's strategies,

- $L(L>1)$ is the spreading gain for the CDMA network which is equal for each user,

- $h_{i}$ is the uplink channel gain of the user $i$,

- $\sigma^{2}$ is the ambient noise in network.

The heterogenous users case gives the following Stackelberg tariff game where

- On the first stage for a fixed tariff the users compete with each other trying to maximize selfishly own payoff where the payoff to user $i$ is given as follows:

$$
v_{U i}(\mathbf{T})=U_{i}\left(R_{i}(\mathbf{T})\right)-C R_{i}(\mathbf{T}),
$$

where $U_{i}$ is the utility of the user $i$ having the same properties as for the one user plot.

Then for a fixed $C$ the equilibrium user's strategies $\mathbf{T}^{*}=\left(T_{1}^{*}, \ldots, T_{N}^{*}\right)$ present a Nash equilibrium, i.e.

$$
v_{U i}\left(T_{i}^{*}, \mathbf{T}_{-i}^{*}\right) \geq v_{U i}\left(T_{i}, \mathbf{T}_{-i}^{*}\right)
$$

for $i \in[1, N]$ and any strategies $\left(T_{1}, \ldots, T_{N}\right)$.

- On the second stage of the game the provider, knowing that the users will be act as it was described on the fist step, assigns tariff $C$ is going to maximize its total profit, i.e. the following payoff:

$$
v_{P}(C)=C \sum_{i=1}^{N} R\left(T_{i}\right)
$$




\subsection{Equilibrium between users}

To find the equilibrium user's strategies on the first step of the game we have to find the derivative of $v_{U i}$ on $T_{i}$ as follows:

$$
\frac{\partial v_{U i}(\mathbf{T})}{\partial T_{i}}=\left(U_{i}^{\prime}\left(R_{i}(\mathbf{T})\right)-C\right) \frac{\partial R_{i}(\mathbf{T})}{\partial T_{i}} .
$$

Then the inside components of equilibrium strategies can be found as a solution of the equations:

$$
L h_{i} T_{i}-D_{i}(C) \sum_{k=1, k \neq i}^{N} h_{k} T_{k}=D_{i}(C) \sigma^{2},
$$

where

$$
D_{i}(C)=e^{\left(U_{i}^{\prime}\right)^{-1}(C)}-1 .
$$

We can rewrite (10) in the following form

$$
h_{i} T_{i}=\frac{D_{i}(C)}{L+D_{i}(C)}\left(\sum_{k=1}^{N} h_{k} T_{k}+\sigma^{2}\right)
$$

We assume that $\left(U_{i}^{\prime}\right)^{-1}(C)=0$ at the same $C=\bar{C}$ how it takes place for example with the shifted $\alpha$-fairness utility. Thus, $T_{i}>0$ for $C \geq \bar{C}$ and we can assume that now $C<\bar{C}$.

Let $I^{r}$ be the set of the inside component of the equilibrium and $r$ is the number of such components. It is clear that $I^{N}=[1, N]$ and $T_{k}(C)=\bar{T}_{k}$ for $k \in[1, N] \backslash I^{r}$.

Summing up all the equations (11) for $i \in I^{r}$ we can find that

$$
\sum_{k \in I^{r}} h_{k} T_{k}=\frac{\sum_{k \in I^{r}} \frac{D_{k}(C)}{L+D_{k}(C)}\left(\sum_{k \in[1, n] \backslash I^{r}} h_{k} \bar{T}_{k}+\sigma^{2}\right)}{1-\sum_{k \in I^{r}} \frac{D_{k}(C)}{L+D_{k}(C)}} .
$$

Substituting (12) into (11) yields that

$$
T_{i}=F_{i}\left(C, I^{r}\right)
$$

with

$$
\begin{aligned}
F_{i}\left(C, I^{r}\right) & =\frac{\sigma^{2}+\sum_{k \in I^{r}} \frac{D_{k}(C)}{L+D_{k}(C)} \sum_{k \in[1, n] \backslash I^{r}} h_{k} \bar{T}_{k}}{1-\sum_{k \in I^{r}} \frac{D_{k}(C)}{L+D_{k}(C)}} \\
& \times \frac{D_{i}(C)}{\left(L+D_{i}(C)\right) h_{i}} .
\end{aligned}
$$

In a particular context in which all users have the same utility function, we are able to obtain a closed form expression of the equilibrium between the users.

THEOREM 5. For a fixed tariff $C$, if all users have the same utility function $U$, the equilibrium users strategies $T_{i}(C)$ for all user $i$ is given by

$$
T_{i}(C)= \begin{cases}\frac{\sigma^{2}+\sum_{j=K+1}^{N} h_{j} \bar{T}_{j}}{h_{i}} & \\ \times \frac{D(C)}{L-(K-1) D(C)}, & i \in[1, K], \\ \bar{T}_{i}, & i \in[K+1, N] .\end{cases}
$$

where $K$ is the unique integer in $\{0, \ldots, N\}$ such that

$$
a_{K+1} \leq e^{\left(U^{\prime}\right)^{-1}(C)}-1<a_{K}
$$

with

$$
a_{k}=\frac{L h_{k} \bar{T}_{k}}{\sigma^{2}+\sum_{j=k+1}^{N} h_{j} \bar{T}_{j}+(k-1) h_{k} \bar{T}_{k}} \text { for } k \in[1, N]
$$

and

$$
a_{0}=\infty \text { and } a_{N+1}=0 .
$$

Proof: In this section we consider the case of equal user utility, so

$$
U_{i}=U
$$

and

$$
D_{i}(C)=D(C):=e^{\left(U^{\prime}\right)^{-1}(C)}-1 \text { for } i \in[1, N] .
$$

Then, (11) is equivalent to

$$
h_{i} T_{i}=\frac{D(C)}{L+D(C)}\left(\sum_{k=1}^{N} h_{k} T_{k}+\sigma^{2}\right) .
$$

Without loss of generality we can assume the users are renumbered in non-increasing order by faded total power, i.e. as follows:

$$
h_{1} \bar{T}_{1} \geq h_{2} \bar{T}_{2} \geq \ldots \geq h_{N} \bar{T}_{N} .
$$

Then by (19) there is a $K \in[1, N]$ such that

$$
T_{i}(C) \begin{cases}\in\left(0, \bar{T}_{i}\right), & i \in[1, K] \\ =\bar{T}_{i} & i \in[K+1, N] .\end{cases}
$$

So, $I^{K}=[1, K]$. Then $(12)$ is equivalent to

$$
\begin{aligned}
& (L-(K-1) D(C)) \sum_{i=1}^{K} h_{i} T_{i}(C) \\
& =K D(C)\left(\sigma^{2}+\sum_{i=K+1}^{N} h_{i} \bar{T}_{i}\right) .
\end{aligned}
$$

Taking into account the fact that $T_{i}$ are non-negative implies that

$$
(K-1) D(C)<L .
$$

The formula (21) jointly with (19) yields that $T_{i}(C)$ has to have the form given by (15).

Now we have to find the switching point $K$. By the assumption (20) it can be found from the following condition

$$
h_{K} \bar{T}_{K} \geq h_{K} T_{K}(C) \text { and } h_{K} T_{K}(C)>h_{K+1} \bar{T}_{K+1} .
$$

Taking into account (15) the last two inequalities turn into the following ones:

$$
\left(\sigma^{2}+\sum_{j=K+1}^{N} h_{j} \bar{T}_{j}\right) \frac{D(C)}{L-(K-1) D(C)}<\bar{T}_{K} h_{K}
$$

and

$$
\left(\sigma^{2}+\sum_{j=K+1}^{N} h_{j} \bar{T}_{j}\right) \frac{D(C)}{L-(K-1) D(C)} \geq \bar{T}_{K+1} h_{K+1} .
$$


The last two conditions are equivalent to the following one for $K \in[0, N]$ :

$$
a_{K+1} \leq D(C)<a_{K}
$$

with sequence $\left\{a_{k}\right\}$ given by (17) and (18).

Also, by (16)

$$
\begin{aligned}
D(C)< & \frac{L h_{K} \bar{T}_{K}}{\sigma^{2}+\sum_{j=k+1}^{N} h_{j} \bar{T}_{j}+(K-1) h_{K} \bar{T}_{K}} \\
= & \frac{L}{K-1+\left(\sigma^{2}+\sum_{j=k+1}^{N} h_{j} \bar{T}_{j}\right) /\left(h_{K} \bar{T}_{K}\right)} \\
& <\frac{L}{K-1} .
\end{aligned}
$$

So, the condition (22) holds and $T_{i}$ given by (15) are positive.

Also, $a_{k}$ is non-increasing sequence since

$$
\begin{aligned}
& a_{k+1}-a_{k}=\frac{L h_{k+1} \bar{T}_{k+1}}{\sigma^{2}+\sum_{j=k+2}^{N} h_{j} \bar{T}_{j}+k h_{k+1} \bar{T}_{k+1}} \\
& -\frac{L h_{k} \bar{T}_{k}}{\sigma^{2}+\sum_{j=k+1}^{N} h_{j} \bar{T}_{j}+(k-1) h_{k} \bar{T}_{k}} \\
& =L \frac{\left(h_{k+1} \bar{T}_{k+1}-h_{k} \bar{T}_{k}\right)\left(\sigma^{2}+\sum_{j=k+2}^{N} h_{j} \bar{T}_{j}\right)}{\sigma^{2}+\sum_{j=k+1}^{N} h_{j} \bar{T}_{j}+(k-1) h_{k} \bar{T}_{k}} \\
& \times \frac{1}{\sigma^{2}+\sum_{j=k+2}^{N} h_{j} \bar{T}_{j}+k h_{k+1} \bar{T}_{k+1}} \leq .
\end{aligned}
$$

Thus, $K$ given by (16) is uniquely defined. This implies the following result describing the equilibrium user strategies given by (15). Where we compute $K \in[0, N]$ using (16) and the sequence $\left\{a_{k}\right\}$ is defined by (17).

\subsection{Discussion: power or throughput pricing?}

It is interesting to note that for power tariff cases studied in [1], [6], the optimal behavior some users or all them at once can be cut off from the network by high tariff. Meanwhile for the throughput tariff model, if only one user cut off from the network than all the users cut off from network. So, in the throughput tariff model providers has more motivation to keep all the available users within its service and do not push them away to switch to the other provider.

Now we answer on the question what makes power and throughput control different. For power control the user's payoff are given as follows:

$$
v_{U i}(\mathbf{T})=R_{i}(\mathbf{T})-C T_{i}
$$

To find the equilibrium user's strategies we have to find the derivative of $v_{U i}$ on $T_{i}$ as follows:

$$
\frac{\partial v_{U i}(\mathbf{T})}{\partial T_{i}}=\frac{L h_{i}}{L h_{i} T_{i}+\sum_{k=1, k \neq i}^{N} h_{k} T_{k}+\sigma^{2}}-C .
$$

Then the inside components of equilibrium strategies can be found as a solution of the equations:

$$
(L-1) h_{i} T_{i}=\frac{L h_{i}}{C}-\sigma^{2}-\sum_{k=1}^{N} h_{k} T_{k} .
$$

It is clear that these equations do not have the nice property of the equations (19) for the throughput control turning all the $T_{i}$ into zero simultaneously. That is why in power control some user's can be cut off from the network meanwhile in throughput control either everybody has access to network or nobody.

\subsection{Optimal tariff for the service provider}

Now turn our attention to the user's throughput $R_{i}(\mathbf{T})$. Since by Theorem 5 we know the user's equilibrium now we find it easily as follows in closed form as follows:

Proposition 1. The optimal throughput are given as follows:

$$
\begin{aligned}
& \text { (a) if } j \in[K+1, N] \text { then } \\
& R_{i}(\boldsymbol{T}) \\
& =\ln \left(1+\frac{L h_{i} \bar{T}_{i}}{\frac{(K+D(C))\left(\sigma^{2}+\sum_{j=K+1}^{N} h_{j} \bar{T}_{j}\right)}{L-(K-1) D(C)}-h_{i} \bar{T}_{i}}\right),
\end{aligned}
$$

(b) if $j \in[1, K]$ then

$$
R_{i}(\boldsymbol{T})=\ln (1+D(C))=\left(U^{\prime}\right)^{-1}(C),
$$

where $K$ is given by (16).

It is interesting that the users $i, i \in[1, K]$ have the same throughput meanwhile the throughput the rest users are different. So, the equilibrium for the user $5 \mathrm{~s}$ with big faded total power $h_{i} \bar{T}_{i}$ is quite fair since all these users have the same throughput. Thus, we have proved the following result supplying the optimal tariff.

THEOREM 6. The optimal tariff of provider in the Stackelberg tariff game with $N$ users is given as follows

$$
C_{*}=\operatorname{argmax}_{C \in[0, \bar{C}]} \Theta(C),
$$

where

$$
\begin{aligned}
& \Theta(C)=C[K(C) \ln (1+D(C)) \\
& +\sum_{i=K(C)+1}^{N} \ln (1 \\
& \left.\left.+\frac{L h_{i} \bar{T}_{i}}{\frac{(K(C)+D(C))\left(\sigma^{2}+\sum_{j=K(C)+1}^{N} h_{j} \bar{T}_{j}\right)}{L-(K(C)-1) D(C)}-h_{i} \bar{T}_{i}}\right)\right],
\end{aligned}
$$

with $K(C) \in[0, N]$ is such that

$$
a_{K(C)+1} \leq D(C)<a_{K(C)},
$$

with sequence $\left\{a_{k}\right\}$ given by (17) and (18). 
Proof. By Theorem 5 the optimal $T_{i}(C)$ has to have the form given by (15) with $K=K(C)$ given by (16), or, what it is equivalent, by (23). Then the result follows from Proposition 1 and the following obvious relation:

$$
v_{P}(C)=C \sum_{i=1}^{K(C)} R_{i}(\mathbf{T})+C \sum_{i=K(C)+1}^{N} R_{i}(\mathbf{T}) .
$$

Given the theorem, we deduce two particular results where first, none users choose to transmit with full power, i.e. $T_{i}(C)<\bar{T}_{i}$ for all $i$, second, where all users transmit with full power, i.e. $T_{i}(C)=\bar{T}_{i}$ for all $i$.

Proposition 2. In particular, we have the two following extreme cases:

(a) if

$$
D(C)<\frac{L h_{N} \bar{T}_{N}}{\sigma^{2}+(N-1) h_{N} \bar{T}_{N}}
$$

then

$$
T_{i}(C)<\bar{T}_{i} \text { for any } i .
$$

So, under condition (24) the user's equilibrium is inside one,

(b) if

$$
\frac{L h_{1} \bar{T}_{1}}{\sigma^{2}+\sum_{j=2}^{N} h_{j} \bar{T}_{j}} \leq D(C)
$$

then

$$
T_{i}(C)=\bar{T}_{i} \text { for any } i .
$$

So, under condition (26) in the equilibrium all the users employ the total power.

Proof. By Theorem 5, (25) holds if and only if $D(C)>$ $a_{N}$ and the last inequality is equivalent to (24). Similarly, (27) holds if and only if $D(C)>a_{N}$ and the last inequality is equivalent to (26).

As a numerical example we considered five users plot $(N=$ 5 ) with shifted $\alpha(\alpha=0.3,1)$-fairness utility and crosstalk coefficient $L=2$, the background noise $\sigma=2$, the same fading gain coefficient $h=(1,1,1,1,1)$ and different total powers $\bar{T}=\left(12,11,10,9, \bar{T}_{5}\right)$ where $\bar{T}_{5} \in[0,5]$. Figure 8 demonstrates how appearing new user (the case $\bar{T}_{5}=0$ is equivalent to the situation where there is only four users) impacts on tariff politics. Of course, this politics also depends on the user's utility.

We have the following result when users are symmetric, i.e. $h_{i}=h$ and $\bar{T}_{i}=\bar{T}$ for all $i$.

THEOREM 7. For symmetrical users (so, if $h_{i}=h$ and $\bar{T}_{i}=\bar{T}$ for any $i$ ) the optimal tariff for the service provider is

$$
C_{*}=\operatorname{argmax}_{C \in\left[C_{0}, 1\right]} C\left(U^{\prime}\right)^{-1}(C) .
$$

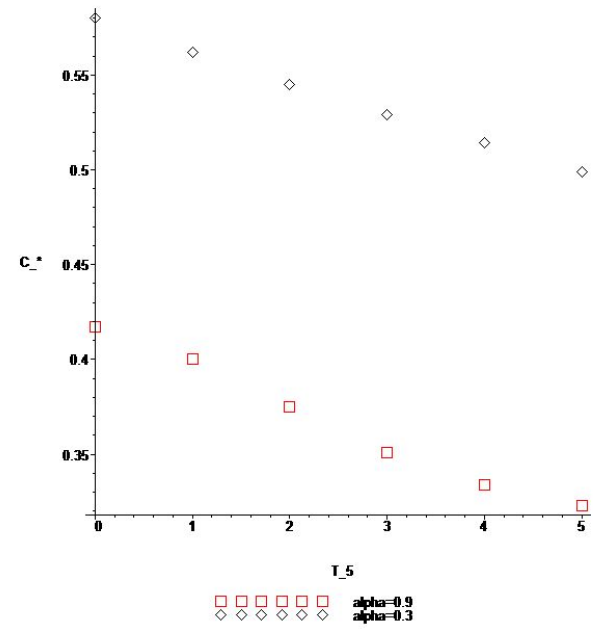

Figure 9: The optimal tariff for $\bar{T}_{5} \in[0,5]$

In particular, for utilities such that $C\left(U^{\prime}\right)^{-1}(C)$ is decreasing in $\left[C_{0}, 1\right]$ how it takes place for utilities given by (2) the optimal tariff is

$$
C_{*}=C_{0}:=D^{-1}\left(\frac{L h \bar{T}}{\sigma^{2}+h(N-1) \bar{T}}\right) .
$$

Then, for $\alpha$-shifted fairness utility the optimal tariff is

$$
C_{*}=\frac{1}{\left(1+\ln \left(1+\frac{L h \bar{T}}{\sigma^{2}+h(N-1) \bar{T}}\right)\right)^{\alpha}},
$$

which brings the following profit to provider

$$
v_{P}=\frac{N \ln \left(1+\frac{L h \bar{T}}{\sigma^{2}+h(N-1) \bar{T}}\right)}{\left(1+\ln \left(1+\frac{L h \bar{T}}{\sigma^{2}+h(N-1) \bar{T}}\right)\right)^{\alpha} .}
$$

Of course the optimal profit of provider is increasing by number of users, but what it tends to $L$ while $N$ is increasing to infinity.

Proof: If all the users equal, namely, $h_{i}=h$ and $\bar{T}_{i}=\bar{T}$ for any $i$ then the optimal strategy $T_{i}(C)$ of user $i$ for a fixed tariff $C$ is given as follows:

$$
T_{i}(C):=T(C)= \begin{cases}\bar{T}, & C \leq C_{0}, \\ \frac{\sigma^{2} D(C)}{h(L-(N-1) D(C))}, & C_{0}<C<1, \\ 0, & 1 \leq C,\end{cases}
$$

where $C_{0}$ is given by (28).

So, the provider's payoff is given as follows:

$$
v_{P}(C)= \begin{cases}C N \ln \left(1+\frac{L h \bar{T}}{\sigma^{2}+(N-1) h \bar{T}}\right), & C \leq C_{0}, \\ N C\left(U^{\prime}\right)^{-1}(C), & C_{0}<C<1, \\ 0, & 1 \leq C .\end{cases}
$$

Since $v_{P}(C)$ is increasing in $\left(0, C_{0}\right)$ and it is constant in $(1, \infty)$ the result follows. 


\section{CONCLUSION}

In this paper we investigate the pricing mechanism with the value of the tariff proportional to the throughput. Our models shows what impact the user's capabilities, network quality and the user's demands have on the tariff formation. We show that the less demandable user rises up tariff and the provider's profit. We also demonstrate that the improving quality of the network puts down tariff and increases the provider's profit. So, investing into new technologies that can improve service are always good for the provider as well as for the user. We show that larger user's capability cuts down tariff and increases the provider's profit. We demonstrate that less demandable user gets more satisfaction from the network service and, of course, this satisfaction is increasing with the network quality and intention to employ it. We demonstrate that the expectation on the increasing user's capability can induce jumping reduction of the tariff and increasing expected provider profit. Meanwhile impairs expectation about increasing in user activity can increase tariff and decrease expected provider profit. Finally, we extend our model to $N$ users case and obtain its solution in closed form. It is interesting to note that for power tariff models [1], [6] lead to inefficient equilibrium behavior of some users. Indeed, some of them at once can be cut off from the network by high tariff meanwhile for the throughput tariff model studied in this paper, it could happen only for all the users at once. So, in the throughput tariff context, providers have more motivation to keep all the available users within its service and do not push them away to switch to the other provider.

Theorem 7 allows to get also quite intriguing result for the shifted $\alpha$-fairness utilities. Since the optimal provider's profit with increasing number of user has a finite limit, namely, $L$ it tells that just attracting new users cannot increase profit to provider essentially. The politics of attracting new users are important, but to get bigger profit it is not enough. To do so providers have to present to users new services users agree to pay for. We are going to investigate this problem in the future. Also we plan to consider multiuser scenario with incomplete information and we plan to extend the current $N$ user model to the case of $S$-form utilities and a general case of non-homogeneous utilities.

\section{REFERENCES}

[1] T. Alpcan, T. Basar, R. Srikant, and E. Altman, CDMA Uplink Power Control as a Noncooperative Game Elsevier Wireless Networks, vol. 8, 2002, pp. 659-670.

[2] E. Altman, K. Avrachenkov, and A. Garnaev, Generalized alpha-fair resource allocation in wireless networks. In Proc The 47th IEEE Conference on Decision and Control (CDC 2008), Cancun, Mexico, 2008, pp. 2414-2419.

[3] E. Altman, K. Avrachenkov and A. Garnaev Taxation for Green Communication WiOpt 2010.

[4] T. Basar, R. Shrikant A Stackelberg Netwoork Game with a Large Number of Followers, JOTA, 115, pp. 479-490.

[5] T. Basar, R. Shrikant Revenue-Maximizimg Pricing and Capacity Expansion in a Many-Users Regime, IEEE Infocom 2002.

[6] A. Al Daoud, T. Alpcan, S. Agarwal and M.Alanyali
A Stackelberg Game for Pricing Uplink Power in Wide-Band Cognitive Radio Networks IEEE CDC 2008.

[7] L. Duan, J. Huang and B. Shou Cognitive Mobile Virtual Network Operator: Investment and Pricing with Supply Uncertainty. Infocom 2010.

[8] A. Garnaev, Y. Hayel, E. Altman, K. Avrachenkov Optimal hierarchical pricing schemes for wireless network usage and resource allocation in proceedings of NETCOOP 2010.

[9] F. Meshkati, H. V. Poor, S. C. Schwartz, and N. B. Mandayam, An energy-efficient approach to power control and receiver design in wireless data networks. IEEE Trans. on Commun., 52, 2005, 1885-1894.

[10] D. Niyato, E. Hossain Optimal Price Competition for Spectrum Sharing in Cognitive Radio: A Dynamic Game-Theoretic Approach. IEEE Globecom 2007.

[11] E.E.Tsiropoulou, T.Kastrinogiannis, and S.Papavassiliou Uplink Power Control in QoS-aware Multi-Service CDMA Wireless Networks. Journal of Communications, 4, 2009, 654-668. 